\title{
A Method for the Automatic Analysis of Colour Category Pixel Shifts During Dichromatic Vision
}

\author{
Mike Bennett and Aaron Quigley \\ Imaging, Visualisation \& Graphics Lab, \\ Systems Research Group, School of Computer Science \& Informatics, \\ University College Dublin, Dublin 4, Ireland \\ \{mike. bennett, aaron.quigley\}@ucd.ie
}

\begin{abstract}
In this paper we present a method for automatically evaluating the amount of colour changes images undergo when perceived by individuals with colour deficient vision. This measure enables the classification of images based on the extent images visually change when viewed by people with one of the three classes of dichromatic (protanopia, deuteranopia, and tritanopia) colour vision. By measuring the extent that colour images appear perceptually different a designer, or automated layout technique, will have an indication of whether the choice of colour usage in an image could lead to colour ambiguity or colour confusions.
\end{abstract}

\section{Introduction}

Colour is widely used in information visualisations, user interfaces, the design of physical spaces, safety systems and in many other areas. When colour is used correctly it enhances people's abilities to carry out tasks, such as distinguishing objects and classifying them into groups (Figure $1^{1}$ ).

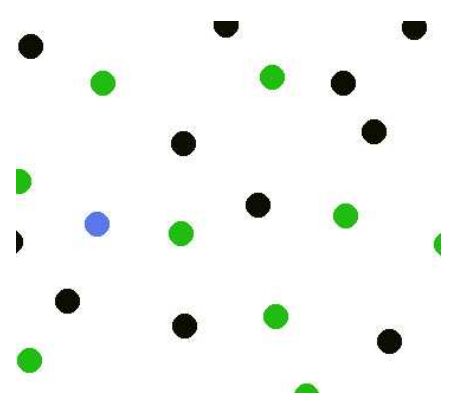

Fig. 1. An example of colour used to create three groups of dots (green, black, blue). Blue is also used to create a visual pop out effect.

\footnotetext{
${ }^{1}$ Colours used in the figures in this paper may not appear correctly when printed.
} 
Yet the ability to perceive colour is something which is often taken for granted. There exists many people for whom trichromatic colour vision is impossible. Colour deficient vision (dyschromatopsia), or colour blindness as it is more commonly known, affects approximately $8 \%$ of males and $0.4 \%$ of females [1][2].

In this paper we present a method for automatically evaluating the amount of colour changes images undergo when perceived by individuals with colour deficient vision (CDV). This measure enables the classification of images based on the extent images visually change when viewed by people with one of the three classes of dichromatic (protanopia, deuteranopia, and tritanopia) colour vision. By measuring the extent that colour images appear perceptually different a designer, or automated layout technique, will have an indication of whether the choice of colour usage in an image could lead to colour ambiguity or colour confusions.

For example, colour confusions can arise if a dichromatic viewer is watching a football game on TV where one team is wearing a red jersey and the other team is wearing a green jersey. Both teams could easily be perceived as wearing the same colour jersey. Depending on the wavelengths of light an individual with protanopia or deuteranopia will not be able to distinguish between red and green. The problem is that red and green become weakly perceptually different. The colours become metamers due to the physiology of CDV eyes. That is, the colours are perceived as belonging to the same colour category [3] and may only vary in saturation and lightness.

In the following sections we provide background information on CDV and related aids for evaluating the impact of CDV. Following this is an outline of our Colour Category Pixel Shifts (CCPS) method, and related Refined Colour Category Pixel Shifts (RCCPS) method, with an elaboration on how both work. We then present the results of an analysis of three hundred natural images processed with CCPS.

\section{Background}

\subsection{Colour Deficient Vision}

A common misconception about people with CDV [4] is that they only see in shades of gray, as though continuously seeing the world as a black-and-white photograph. This is not the case, the vast majority of people with CDV perceive a range of colours.

The human eye consists of approximately 6 to 7 million cones and 120 million rods [5] [6]. There are known to be three classes of cones, each of which has a peak sensitivity to a different wavelength of light. One way of thinking of these classes of cones is that they classify light into three frequency ranges which correspond to the colours perceived as red, green and blue. Rods enable humans to see at night and contribute little to colour vision. Rods are a lot more sensitive to light and are somewhat overwhelmed in normal daylight. Thus cones enable photopic (colour) vision and rods enable scotopic (low light) vision. 
Colour vision deficiencies are the result of a reduced light sensitivity of the cones or a complete lack of one or more classes of the cones. When there is a reduced sensitivity in one class of cones a person is said to have anomalous trichromatic colour vision. When one class of cone is completely missing, or nonfunctioning, a person has dichromatic vision. Cone monochromacy is when only one class of cones are functioning, and if no cones are functioning or present a person has achromatic vision.

Inherited variations in colour vision cannot currently be 'fixed'. These variations have a physiological basis, which is most often associated with $\mathrm{X}$ chromosome genes. Colour vision deficiencies can be acquired due to the normal aging process and may also develop due to a range of illnesses, such as cataracts, diabetes and age-related macular degeneration.

\subsection{Related Work}

A number of approaches have been taken to helping designers understand, evaluate and reduce the impact of CDV.

One approach to helping people understand the impact of CDV is by providing tools that simulate various kinds of colour defective vision [7][8][9]. These tools can be used by visual designers to evaluate designs. Designers can 'see' the visual designs through another's eyes. For example the Aging Vision Simulator [10] transforms images based on an approximation of how the lens in the human eye develops a yellow tint as people get older. Another example is a colour blindness simulator called Vischeck [9], which we used as part of testing the CCPS and RCCPS methods (Section 3.2). Vischeck transforms the colour in images based on a human visual system model [8] of how dichromatic perceivers see colours.

Examples of approaches for automatically reducing the impact of CDV are the Daltonize algorithm [11] and the work of Rasche et al [12]. In both cases automatic adjustments are made to how colours are used in images. With the Daltonize algorithm visual information which would be hidden from red-green dichromats becomes perceivable. The Daltonization algorithm works by establishing the amount of information in an image that is encoded with red-green colours. Based on this analysis it attempts to shift the red-green colour dimension into the blue-yellow and light-dark dimensions. With colour shifting colours that were distinct could start to become similar, thus introducing colour confusions and ambiguity. Colour constancy over multiple images may also become an issue, i.e. red could be converted into a light blue in one image and into a dark purple in another image.

\section{Colour Category Pixel Shifts}

\subsection{Overview}

CCPS is a measure of the percentage of pixels in an image that change colour category when viewed by individuals with dichromatic colour vision. RCCPS is a measure of how many pixels change colour category in each colour category. 
CCPS provides an overview of how much pixel change occurs, RCCPS provides details about which colour categories undergo changes along with how much change per category.

Both the CCPS and RCCPS measures rely on image transforms as part of the process of generating the measures. Before detailing how CCPS and RCCPS are calculated (Section 3.4) we outline the two key transforms.

The first transform converts an image into an approximation [8] of what an individual with dichromatic CDV would see (Section 3.2). The second transform is a form of colour image segmentation where pixels in an image are grouped according to colour category (Section 3.3).

\subsection{Modeling Dichromatic Colour Vision}

In vision science there are numerous models of different functions in the human visual system [13] but there are a limited number of models of CDV. Existing models [8][9] tend to focus on dichromatic CDV, with an emphasis on protanope and deuteranope vision.

We utilised the Vischeck plugin [9] for Adobe Photoshop to simulate CDV. Three hundred images from the Berkeley Segmentation Data Set (Section 4) were transformed with the plugin. These images where then used for testing CCPS and RCCPS.
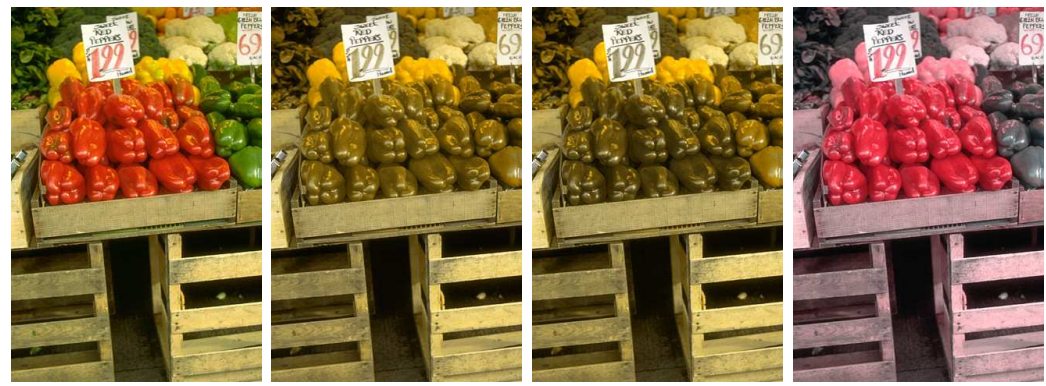

Fig. 2. First image unchanged from [14]. Second (protanope), third (deuteranope) and fourth (tritanope) images were generated with the Vischeck colour blindness simulator.

Each image was transformed into three new images, each of which approximates how a protanope, deuteranope and tritanope perceives the image (Figure 2 ). When more diverse and accurate models of each of the other sub-types of CDV are developed they can also be used to carry out the image transforms.

\subsection{Colour Category Classification of Pixels}

Classifying pixels into perceptual colour categories [15] is a non-trivial task. There is a considerable body of work in both human and automated colour 
category naming and identification [3][16][17]. Based on the findings in colour category theory we partitioned the HSL colour space into the colour categories of White, Black, Red, Green, Blue, Yellow, Gray, Pink, Purple, Orange and Brown.

There are indications that these eleven colour categories tend to be commonly shared worldwide across languages and cultures [18][3] - though there are many unresolved questions about the number and nature of colour categories [19]. As algorithms for perceptually classifying pixels improve they can be utilised in generating the CCPS and RCCPS measures.

When classifying a pixel by colour category it is converted from the RGB to the HSL colour space. Then predominately based on Hue a pixel is classified into one of the eleven colour categories. Saturation and Lightness are used for classifying pixels when they are near their upper and lower bounds, corresponding to Black and White. Brown and orange were treated as a single colour category because distinguishing them often led to pixels which were brown getting classed as orange, and vise versa.

For example, if a pixel is light or dark red it is changed into a single RGB value representing both variations of red. This can result in images getting partially (depending on image complexity) segmented into blocks of colour, i.e. a blue sky with a gradient from light to dark blue becomes a single block of blue.

\subsection{Calculating CCPS \& RCCPS}

A CCPS measure is generated by taking the following steps:

1. Transform an image into that which a dichromatic viewer would perceive (Section 3.2).

2. Classify each pixel in both the original and transformed images into one of the colour categories (Section 3.3).

3. Compare the original and transformed images checking whether each pixel has switched colour category (Section 3.4).

4. Calculate a percentage measure (Eqn. 1) based on the number of pixels which have switched colour categories divided by the total number of pixels in the original image (Section 3.4).

$$
\text { CCPS }=\frac{\text { Shifted Pixels }}{\text { Total Pixels }} * 100
$$

It is possible to obtain a refined breakdown of the single percentage indicator generated by CCPS. This provides more information about how the pixels are shifting colour categories. To get a more detailed breakdown the amount of pixels in each colour category is calculated (Eqn. 2) in both the original and dichromatic transformed image. The difference in the change in the number of pixels per colour category is then used to generate a percentage indicator:

$$
R C C P S=\frac{(C P O-C P T)}{\text { Total Pixels }} * 100
$$


RCCPS $=$ Refined CCPS, CPO = Count of pixels in a specific colour category in the original image, $\mathrm{CPT}=$ Count of pixels in a specific colour category in the transformed image, Total Pixels $=$ Count of total number of pixels in the original image.

Rather than producing a single CCPS value RCCPS is used to obtain a positive or negative percentage indicating how many pixels shifted away from or into a specific colour category. RCCPS can be applied to each colour category, producing a set of numbers that provide detail on how the pixels in each colour category are affected.

For example imagine that CCPS was integrated in a tool for designing user interfaces and that a designer uses CCPS to analyse an image consisting of 50000 pixels. After applying the dichromatic image transform and counting the number of pixels which shifted categories it is found that $42 \%$ of the pixels shifted colour category. A $42 \%$ change sounds serious, but when the designer visually examines the image they establish that most of the pixels which switched category changed from a light gray to a white. If RCCPS was carried out on the image, rather than visually examining it, it would tell the designer that pixels in the gray colour category suddenly decreased while pixels in the white colour category suddenly increased.

The ability to distinguish which colour categories are most affected is important because the three classes of dichromatic CDV are more sensitive to the effects of specific colours switching colour categories, i.e. red or green pixels switching colour categories for protanopes and deuteranopes.

\section{Evaluating the Berkeley Segmentation Data Set}

In this section we present the results of using CCPS to analyse 300 images. The images come from the Berkeley Segmentation Data Set (BSDS) [14], which is "a large data set of natural images that have been segmented by human observers". BSDS was originally created for comparing the performance of image segmentation algorithms.
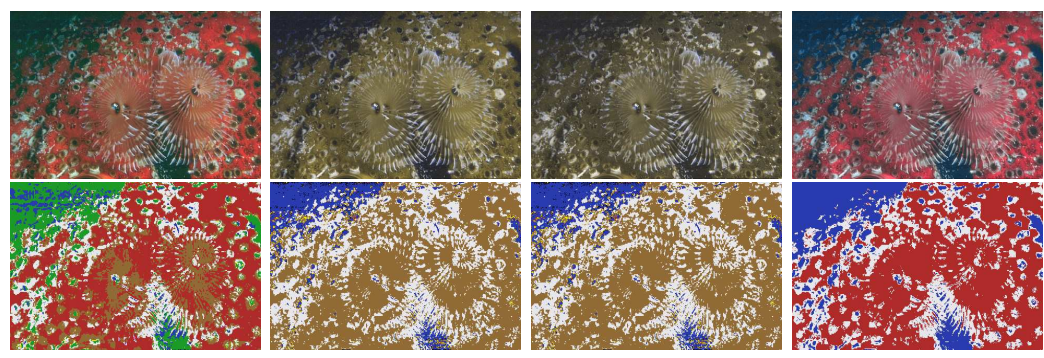

Fig. 3. First row is an example of an original, protanope, deuteranope and tritanope image. The second row shows the images after the pixels have been classified by colour category. 
Each image from BSDS was converted into three further images - a protanope, deuteranope and tritanope image (Figure 3). Then a C++ implementation of CCPS and RCCPS was used to process the 1200 images. The original images had to be processed (Section 3.4, Step 2) to work out the original number of pixels in each colour category in each image. Figure 3 shows an example of an original, protanope, deuteranope and tritanope image where the pixels have been classified by colour category.

After running CCPS on all the images we graphed the results (Figure 4 and Figure 5). One important aspect of the results is that there were a large number of pixels classified into the black, white and gray categories. This was primarily an artifact of how pixels are classified into colour categories. Distinguishing a light gray from a light blue is non-trivial so we errored on the side of caution by treating light colours as gray, very light colours as white and very dark colours as black. This had the effect that colour category pixel shifts tended to be weighted towards colours that were darker and more saturated. An emphasis was placed on pixels for which colour category shifts would be more perceptually distinct.

\section{Rate of Colour Category Pixel Shifts}

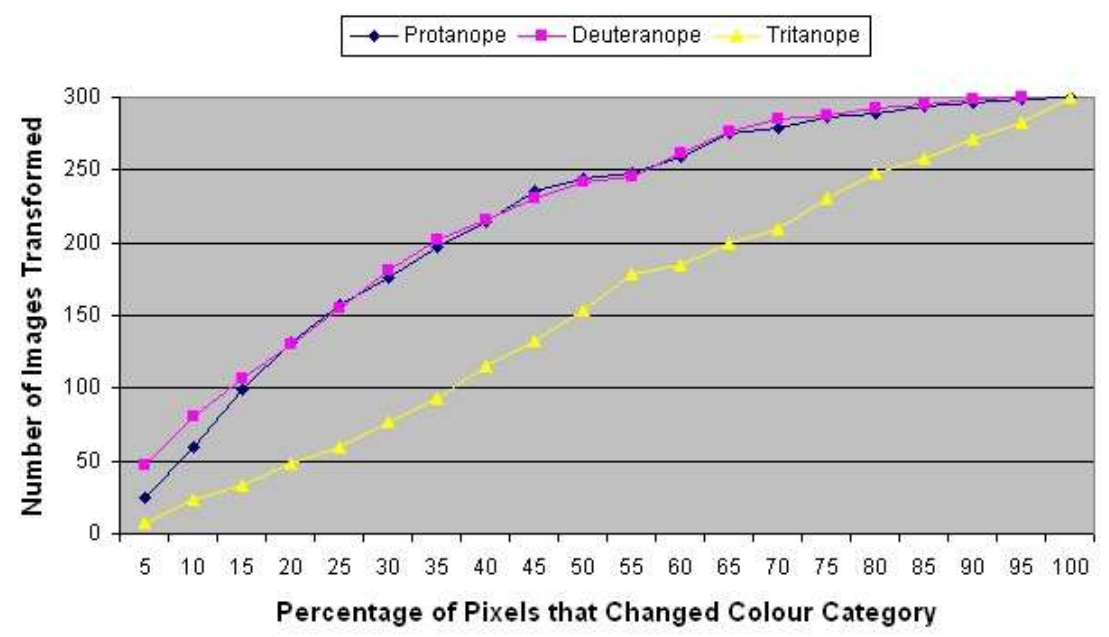

Fig. 4. Rate that CCPS pixels switch colour categories.

Looking at Figure 4 we can see that for protanopes and deuteranopes 100 of the images undergo a $15 \%$ pixel change or less, and that 150 of the images undergo less than a $25 \%$ change in pixel colour catgories. The graph also shows the rate of colour category shifts for deuteranopes and tritanopes. This graph indicates the BSDS images are most likely to undergo change when viewed by tritanopes. 


\section{Distribution of Colour Category Pixel Shifts}

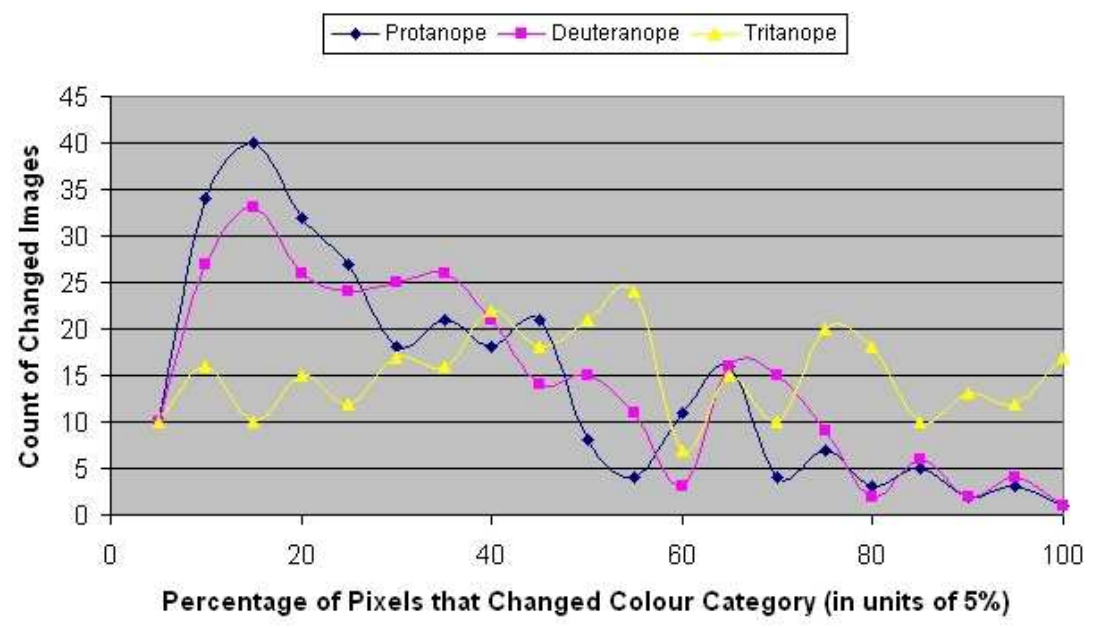

Fig. 5. The distribution of the CCPS percentage of pixels that switched colour categories in the images.

Figure 5 provides a related graph that shows the distribution of percentage changes in colour categories. From this we can see that for protanopes 40 images have colour category pixel changes in the $10 \%$ to $15 \%$ range, and for deuteranopes 33 images have colour category pixel changes in the $10 \%$ to $15 \%$ range. The distribution for tritanopia is extremely varied - this may be due to a number of factors, ranging from the amount of yellow and blue in the BSDS images (a lot are in outdoor settings) to the underlying model used to model tritanopia vision. A more varied image set is required to analyse the interplay between CCPS and tritanopia.

It is important to note that until more refined models of CDV are developed, and very accurate ways of classifying the colour category of pixels become available, CCPS and RCCPS should be treated as heuristic indicators. The results in the graphs should not be interpreted as definitive measures of colour category changes perceived in the world by individuals with dichromatic CDV.

\section{Conclusions}

In this paper we have presented two useful methods, CCPS and RCCPS, for automatically evaluating colour category changes images undergo when perceived by individuals with colour defective vision. CCPS provides an overview of how much colour category pixel change occurs in an image, while RCCPS provides details about which colour categories undergo changes along with how much change occurs per category. 
We implemented CCPS and RCCPS and demonstrated how CCPS provides a measure of colour category changes in images due to dichromatic CDV. This was demonstrated by using CCPS to analyse a collection of images from a publicly available data set.

The CCPS and RCCPS methods could easily be implemented as part of an interface or information visualisation design tool. With the potential benefit of helping designers become aware of how they use colour, while also helping them understand the impact inappropriate colour usage can have on individuals with CDV.

\subsection{Future Work}

This paper has highlighted a number of areas where further research could be carried out. For example research could be focused on developing human visual system models of anomalous trichromatic colour deficient vision. These models could then be used in conjunction with CCPS and RCCPS.

Pixel level colour category changes are a useful measure but it might prove more beneficial to decompose an image into higher-order objects and measure whether these objects undergo colour category changes. Related to this is a method for measuring whether a pixel shifts colour categories such that it becomes the same colour as the pixels surrounding it. This would indicate whether foreground and background colour differences are reduced in images for CDV perceivers.

Finally, better techniques for classifying pixels according to the colour categories they belong to would improve the performance of CCPS and RCCPS.

Acknowledgments This work would not have been possible without the ongoing support from the EU Marie Curie CoViAn project, the IBM Dublin Center for Advanced Studies and the School of Computer Science and Informatics UCD, Ireland.

\section{References}

1. Hunt, R.: Measuring Colour. 3rd edn. Fountain Press (2001)

2. Hurvich, L.M.: Color Vision. Sinauer Associates Inc. (1981)

3. Berlin, B., Kay, P.: Basic Color Terms: Their Universality and Evolution. London: University of California Press (1969)

4. Mollon, J.D., Pokorny, J., Knoblauch, K.: Normal and Defective Colour Vision. Oxford University Press (2003)

5. Bruce, V., Green, P.R., Georgeson, M.A.: Visual Perception: Physiology, Psychology and Ecology. Hove: Psychology (1996)

6. Palmer, S.E.: Vision Science: Photons to Phenomenlogy. MIT Press (1999)

7. Meyer, G., Greenberg, D.: Color-defective vision and computer graphics displays. IEEE Computer Graphics and Applications 8 (1988) 28-40

8. Brettel, H., Viénot, F., Mollon, J.D.: Computerized simulation of color appearance for dichromats. Journal of the Optical Society of America. A, Optics and image science 14 (1997) 2647-2655 
9. Dougherty, R., Wade, A.: Vischeck, http://www.vischeck.com (2006)

10. Becker, S., Tu, C., Kim, H.: Software tools for the effective use of color in web designs. In: Proceedings of the 2004 National Conference on Digital Government Research, Seattle, Washington (2004) 385-386

11. Dougherty, R., Wade, A.: Daltonize algorithm, http://www.vischeck.com/daltonize (2006)

12. Rasche, K., Geist, R., Westall, J.: Re-coloring images for gamuts of lower dimension. In Alexa, M., Marks, J., eds.: Eurographics. Volume 24., The Eurographics Association, Blackwell Publishing (2005)

13. Carney, T., Klein, S.A., Tyler, C.W., Silverstein, A.D., Beutter, B., Levi, D., Watson, A.B., Reeves, A.J., Norcia, A.M., Chen, C.C., Makous, W., Eckstein, M.P.: The development of an image/threshold database for designing and testing human vision models. In: Human Vision, Visual Processing, and Digital Display IX, Proc. SPIE. Volume 3644. (1999) 542-551

14. Martin, D., Fowlkes, C., Tal, D., Malik, J.: A database of human segmented natural images and its application to evaluating segmentation algorithms and measuring ecological statistics. In: Proceedings of the 8th Int'l Conf. Computer Vision. Volume 2. (2001) 416-423

15. Kay, P.: Color categorization. MIT Encyclopedia of the cognitive sciences (1999)

16. Lammens, J.M.G.: A Computational Model of Color Perception and Color Naming. PhD thesis, State University of New York at Buffalo (Computer Science) (1994)

17. Belpaeme, T.: Factors influencing the origins of colour categories. PhD thesis, Artificial Intelligence Lab, Vrije Universiteit Brussel (2002)

18. Yendrikhovskij, S.N.: Computing color categories from statistics of natural images. Journal of Imaging Science and Technology 45 (2001) 409-417

19. Saunders, B., van Brakel, J.: Are there non-trivial constraints on colour categorization? Behavioral and Brain Sciences 20 (1997) 167-228 